\title{
Impact of telemedicine interventions on mortality in patients with acute myocardial infarction: a systematic review and meta-analysis
}

\author{
Milena Soriano Marcolino, ${ }^{1}$ Luciana Marques Maia, ${ }^{1}$ João Antonio Queiroz Oliveira, ${ }^{1}$ \\ Laura Defensor Ribeiro Melo, ${ }^{1}$ Bruno Leonardo Duarte Pereira, ${ }^{1}$ \\ Diomildo Ferreira Andrade-Junior, ${ }^{1}$ Eric Boersma, ${ }^{2}$ Antonio Luiz Ribeiro ${ }^{1}$
}

\begin{abstract}
- Additional material is published online only. To view please visit the journal online (http://dx.doi.org/10.1136/ heartjnl-2018-314539).
\end{abstract}

'Medical School and University Hospital, Universidade Federal de Minas Gerais, Belo Horizonte, Brazil

${ }^{2}$ Department of Cardiology, Thoraxcenter, Erasmus MC Rotterdam, The Netherlands

Correspondence to Dr Milena Soriano Marcolino, Internal Medicine, Universidade Federal de Minas Gerais, Belo Horizonte 30130100, Brazil; milenamarc@gmail.com

Received 26 November 2018 Revised 14 April 2019 Accepted 19 April 2019 Published Online First 28 June 2019

\section{Linked}

- http://dx.doi.org/10.1136/ heartjnl-2019-315278

Check for updates

(C) Author(s) (or their employer(s)) 2019. No commercial re-use. See rights and permissions. Published by BMJ.

To cite: Marcolino MS Maia LM, Oliveira JAQ, et al. Heart 2019:105:1479-1486.

\section{ABSTRACT}

Background Despite the promise of telemedicine to improve care for ischaemic heart disease, there are significant obstacles to implementation. Demonstrating improvement in patient-centred outcomes is important to support development of these innovative strategies.

Objective To assess the impact of telemedicine interventions on mortality after acute myocardial infarction (AMI).

Methods Articles were searched in MEDLINE, Cochrane Central Register of Controlled Trials, Literatura Latino-Americana e do Caribe em Ciências da Saúde (LILACS), Base de Dados de Enfermagem (BDENF), Indice Bibliográfico Español en Ciencias de la Salud (IBECS), Web of Science, Scopus and Google Scholar, from January 2004 to January 2018. Study selection and data extraction were performed by two independent reviewers. In-hospital mortality (primary outcome), and door-to-balloon (DTB) time, 30-day mortality and longterm mortality (secondary outcomes) were assessed. Random effects models were applied to estimate pooled results.

Results Thirty non-randomised controlled and seven quasi-experimental studies were included (16960 patients). They were classified as moderate or serious risk of bias by ROBINS-I (Risk Of Bias In Non-randomized Studies-of Interventions tool). In 31 studies, the intervention was prehospital ECG transmission. Telemedicine was associated with reduced in-hospital mortality compared with usual care (relative risk (RR) $0.63(95 \%$ confidence interval[Cl] 0.55 to 0.72$) ; I^{2}$ $<0.001 \%$ ). DTB time was consistently reduced (mean difference $-28(95 \% \mathrm{Cl}-35$ to -20$) \mathrm{min})$, but showed large heterogeneity $\left(\mathrm{I}^{2}=94 \%\right)$. Thirty-day mortality (RR $0.62 ; 95 \% \mathrm{Cl} 0.43$ to 0.85$)$ and long-term mortality (RR $0.61(95 \% \mathrm{Cl} 0.40$ to 0.92$))$ were also reduced, with moderate heterogeneity $\left(\mathrm{I}^{2}=52 \%\right)$.

Conclusions There is moderate-quality evidence that telemedicine strategies, in particular ECG transmission, combined with the usual care for AMI are associated with reduced in-hospital mortality and very-low quality evidence that they reduce DTB time, 30-day mortality and long-term mortality.

\section{INTRODUCTION}

In an effort to improve outcomes for ischaemic heart disease, innovative strategies are required. ${ }^{12}$ Telemedicine is defined as 'the delivery of health care services (...) by all healthcare professionals using information and communication technologies for the exchange of valid information for diagnosis, treatment and prevention of disease and injuries, research and evaluation, and for the continuing education of health care providers (...)' ${ }^{3}$ It can help to address major challenges faced by healthcare systems, ${ }^{4}$ and it has been used in several healthcare fields. Cardiology is a promising area in telemedicine and, within cardiology, acute coronary syndrome (ACS) care is one of the fields where most of the efforts have been made. ${ }^{5}$

In this context, telemedicine strategies have the potential to improve diagnosis and to increase healthcare professionals' adherence to the therapeutic measures established for ACS, thus improving ACS care. The current evidence has largely been centred on the validation of technologies, feasibility, usability or the impact on process outcomes, but there are paucity of data on the impact on patient-centred outcomes to justify implementation of telemedicine. ${ }^{6}$ Given the challenges to implementing telemedicine systems, benefits should be clearly demonstrated in terms of these patient-centred outcomes. Individual studies are underpowered to detect differences in mortality. Therefore, a systematic review and meta-analysis investigating the theme is warranted and timely. Accordingly, here we present a systematic review and meta-analysis of studies assessing the impact of telemedicine interventions combined with usual care compared with usual care alone on the mortality and time to intervention from acute myocardial infarction (AMI).

\section{METHODS}

Systematic review and meta-analysis methods followed guidance from the Centre for Reviews and Dissemination (CRD), Meta-analysis of Observational Studies in Epidemiology group guidelines and the Cochrane Collaboration. ${ }^{7-9}$ It is reported according to the Preferred Reporting Items for Systematic Review and Meta-Analyses statement. ${ }^{10}$ The investigators registered the protocol with the International Prospective Register of Systematic Reviews (CRD42016025404). 


\section{Data sources and searches}

A literature search, limited to English, French, Dutch, German, Italian, Spanish and Portuguese, was performed using MEDLINE, Cochrane Central Register of Controlled Trials, Scopus, Web of Science, Literatura Latino-Americana e do Caribe em Ciências da Saúde (LILACS), Base de Dados de Enfermagem (BDENF), Indice Bibliográfico Español en Ciencias de la Salud (IBECs) to identify relevant studies published from January 2004 to January 2018. Google Scholar was used as a grey literature source, and the search was limited to English, Spanish and Portuguese.

For MEDLINE, combinations of the MESH terms telemedicine, reminder systems, remote consultation, videoconferencing, clinical decision support systems, computer-assisted decision making, computer assisted decision making, acute coronary syndrome, angina pectoris, coronary occlusion, coronary thrombosis, myocardial infarction and chest pain, and the terms telemonitoring, transtelephonic, telehealthcare, teleconsultation, telecardiology, tele ecg and tele-eletrocardiography were used. In the other databases, different combinations of the same terms and the keywords mobile health, telehealth, health information technology were used. Searches on Google Scholar used the terms myocardial infarction, chest pain, angina, acute coronary syndrome, telemedicine, remote consultation, telehealth, reminder systems, video conference, mobile health and $e$-Health.

Additional publications were identified by hand-searching reference lists of included studies, reviews and meta-analyses.

\section{Study selection}

Studies were included if they had a comparative study design and compared any strategy of telemedicine combined with usual care versus usual care alone for the diagnosis and management of patients with AMI (ST elevation myocardial infarction (STEMI) and non-ST elevated myocardial infarction (non-STEMI)). Primary outcome was in-hospital mortality, and secondary outcomes were 30-day mortality, long-term mortality (defined as follow-up time of at least 1 year) and doorto-balloon (DTB) time.

The strategies of telemedicine included in this review were computerised systems for information exchange, electrocardiogram (ECG) recording (prehospital, in a non-percutaneous coronary intervention (PCI) hospital or in-hospital in the emergency department (ED)) and transmission to a cardiac receiving centre, teleconsultation, and exchange of patient data or information via telephone, other mobile devices, short message service or through the internet. If we identified that more than one telemedicine strategy was used, even if the authors of the original study did not report that, this was included in the summary table. Teleconsultation may be defined as 'consultation (...) between distant healthcare professionals with local healthcare professionals concerning a patient's diagnosis and treatment, using telecommunication and information technology to bridge the spatial and functional distance between the participants'. ${ }^{11}$

Exclusion criteria were as follows: (1) studies regarding automatic ECG interpretation without transmission for remote evaluation; (2) studies on chronic coronary disease; (3) studies in which the telemedicine intervention was used after patient discharge; (4) duplicate publications or substudies of included studies. In the final case, the publication with the largest sample and longer follow-up was chosen, unless the publications referred to different follow-up times.

Titles and abstracts were independently screened by two investigators. Articles deemed potentially eligible by either reviewer were retrieved for full-text review. Disagreements were subsequently resolved by a third reviewer.

Corresponding authors were contacted as needed to obtain data not included in the published report. There were at least three contact attempts, using email and Research Gate (www. researchgate.net). In the absence of a response in cases where data were insufficient for analysis, the article was excluded.

\section{Data extraction and quality assessment}

Data were extracted from each study independently by the two investigators, according to a standardised protocol. The third reviewer resolved any disagreements.

No randomised controlled trials matching the inclusion criteria were found. Methodological quality of non-randomised controlled studies was assessed using the Risk Of Bias In Non-randomized Studies-of Interventions tool (ROBINS-I). ${ }^{12}$ ROBINS-I was specifically developed for studies with a cohort type of design, in which individuals who have received (or are receiving) different interventions are followed up over time, and a specific version of the tool for quasi-experimental (before and after) studies and studies with historical control has not been published yet. Therefore, for these studies we decided a priori to classify the risk of bias due to confounding as serious, as there is evidence of a general trend in reduced mortality and lower DTB times for patients with myocardial infarction over time. ${ }^{13}$ Each trial was independently scored by two investigators and any disagreements were solved by consensus.

The overall quality of the body of evidence was rated by using the Grading of Recommendations Assessment, Development and Evaluation (GRADE) approach. ${ }^{14}$

\section{Data synthesis and analyses}

With regards to DTB time, when mean and standard deviation (SD) were not available, the authors were contacted. In the case of unavailability of the requested information, mean and variance were estimated by using the median, interquartile range (IQR), sample size and/or reported CIs. ${ }^{15}$ In studies in which there was more than one control group, the group that was more similar to the intervention group (except for the intervention) was selected for inclusion in the meta-analysis. For example, in studies with one intervention group with prehospital ECG transmission and two control groups: (1) if in one control group the diagnosis was prehospital and the other one in-hospital, the first one was selected ${ }^{16}$; (ii) if in one control group patients were admitted to the same hospital as the intervention group, and in the other control group patients were admitted to non-PCI capable hospital or community hospitals, only the first control group was selected. ${ }^{17} 18$ In case of studies with historical and concurrent controls, the concurrent controls were chosen. ${ }^{19-21}$ If there was more than one intervention group, the weighted mean and weighted SD of mean time of reperfusion was calculated and used in the meta-analysis.

All analyses were performed using the Comprehensive Meta-Analysis Software v. 3.3.070 (CMA). A random effects model was used for pooling the results of included studies, as clinical heterogeneity was expected. The software calculated mean differences for continuous outcomes; pooled relative risks (RRs) and pooled risk difference for binary outcomes, and calculated 95\% CIs and two-sided P values for each outcome. Number needed to treat (NNT) was calculated as 1 /pooled risk difference. Statistical heterogeneity of the treatment effect among studies was investigated by using Cochran $Q$ test, $\chi^{2}$ statistic and $\mathrm{I}^{2}$ statistic, in which values greater than $50 \%$ were considered 
indicative of high heterogeneity. A p value $<0.05$ (two sided) was considered statistically significant.

A priori, we specified the following possible explanations for heterogeneity: study design, specific features of each local healthcare system, jurisdiction size, and specificities of the telemedicine intervention.

Sensitivity and subgroup analyses were prespecified and performed only for the primary outcome. In sensitivity analyses, the effect size was examined by omitting studies individually and also excluding simultaneously studies with extreme results (highest and lowest impact), those with the biggest losses and the two studies authored by our group. Subgroup analyses were performed to assess whether there was a difference in results for in-hospital mortality with respect to the study design and AMI reperfusion type (thrombolysis or PCI).

A funnel plot was constructed to assess the possibility of publication bias. ${ }^{22}$ The symmetry of the plot was evaluated both visually and formally with Egger's test. The implications for our results were assessed by the fail-safe $\mathrm{N}$ and the trim-and-fill method. ${ }^{23}$

\section{RESULTS}

\section{Search results}

Of the 8980 articles retrieved, 4168 were from databases (3064 MEDLINE, 113 LILACS, 178 Cochrane Trials, 2284 Scopus, 351 Web of Science), 2955 from Google Scholar and 35 other articles were identified from the references of evaluated studies. After duplicates were removed, there were 8120 unique citations. Of these, 7993 were excluded after title and/or abstract analysis.

Therefore, 127 full-text articles were assessed for eligibility. Of these, 92 did not meet the inclusion criteria and were excluded. Additional six studies had incomplete data such that the analysis was not possible and the authors did not respond to any contact attempts and thus were excluded. Two articles from a single study were included, as they assessed mortality in different follow-up periods. Thus, the final number of included studies was 35 (36 articles). A flow diagram of literature search and study selection is shown in figure 1 .

\section{Study and patient characteristics}

The main characteristics of the included studies are summarised in online supplementary table S1, and their references are provided in the online supplementary file. Eight studies took place in North America (7 in the United States and 1 in Canada), 11 in Europe (4 in Denmark, 5 in Italy, 1 in Hungary and 1 in the Netherlands), 9 in Asia (3 in Japan, 2 in Korea, 1 in China, 1 in Singapore, 1 in Taiwan and 1 in Turkey), 2 in Australia and 5 in South America (all of them in Brazil). One of those studies was published in two different articles, for short and long-term follow-up. ${ }^{24}{ }^{25}$ Funding information is given in the online supplementary file.

Included studies provided a total of 16763 patients, of which 7897 in the telemedicine strategies group and 8866 in usual care group. Overall, $69.1 \%$ were male. Twenty-eight studies were non-randomised controlled, of which 22 had a concurrent control group, 3 had historical and concurrent control groups and 3 had historical control group. The remaining seven studies had quasi-experimental design. All studies included patients with STEMI, four of them included also patients with non-STEMI. Recruitment time was 20 years, from 1995 to 2015. In all studies, the ECG transmission was the strategy used. Fourteen of them used this strategy in combination with teleconsultation and in 31 the intervention was prehospital ECG transmission. With regards to the studies which included exclusively patients with STEMI, in 8 studies, the intervention was combined with catheterisation laboratory activation bypassing the ED for all patients; in 6 studies there was no ED bypass; in 6 studies it was heterogeneous; and in 11 studies it was not clear (online supplementary table S1).

Online supplementary tables S1 and S3 show the risk of bias assessment using ROBINS-I. For the primary outcome, eight studies had serious risk of bias, nine had moderate risk and one had low risk. For 30-day mortality, two studies had serious risk of bias, two had moderate risk and one had low risk. For longterm mortality, five studies had serious risk of bias, one had moderate risk and one had low risk. For DTB time, 6 studies had serious risk of bias, 17 had moderate risk and 3 had low risk.

\section{Primary and secondary outcomes}

Table 1 is the summary of findings (SOFs) table with the GRADE classification of the quality of evidence. Telemedicine combined with usual care was significantly associated to reduced in-hospital mortality: $4.9 \%$ vs $8.4 \%$ (figure 2 ). There was no evidence of heterogeneity $\left(\mathrm{I}^{2}<0.001 \%\right)$. Visual inspection of the funnel plot (figure 3), the 'Trim and fill' method and Egger's statistic $(p=0.55)$ showed no evidence of publication bias.

The subgroup of patients treated with primary PCI included 10 studies (3174 patients). Telemedicine was associated with a statistically significant reduction in in-hospital mortality in this subgroup: $3.0 \%$ vs $5.7 \%$; RR 0.48 (95\% CI 0.33 to 0.71 ). When analysing the other seven studies, in which thrombolysis was used alone, associated with PCI (pharmacoinvasive strategy), or as an alternative to primary PCI in some cases (6468 patients), telemedicine strategies were also beneficial in reducing in-hospital mortality: $7.7 \%$ vs $13.3 \%$; RR 0.65 (95\% CI 0.56 to $0.75)$. There was no evidence of heterogeneity in both analyses $\left(\mathrm{I}^{2}<0.01 \%\right)$ (online supplementary figure $\mathrm{S} 1$ ).

Results of sensitivity analysis are commented in the online supplementary file.

The intervention also showed benefit in 30-day mortality (figure 4A) and long-term mortality (figure 4B), both with moderate heterogeneity $\left(\mathrm{I}^{2}=52 \%\right.$ and $54 \%$, respectively $)$ (table 1).

Among the 26 studies reporting DTB time, 3 provided only the median times or graphic representation of the results (references shown in the online supplementary file). The authors did not respond to contact or did not have the needed data, so those articles were excluded from the meta-analysis. Therefore, 23 studies (7395 patients) were included in the analysis of this outcome (figure 5). The large heterogeneity observed $\left(\mathrm{I}^{2}=94 \%\right)$ reduced confidence in pooled quantitative analysis. Funnel plot inspection and the 'Trim and fill' method suggested low risk of publication bias (online supplementary figure S3), but the Egger's regression test indicated there was a risk of publication bias (one-tailed $\mathrm{p}$ value $=0.377$ ).

\section{DISCUSSION}

We conducted a comprehensive search on the impact of telemedicine for the management of patients with AMI, and observed that these interventions, in particular ECG transmission, were consistently associated with improved AMI survival. Despite the non-randomised design and moderate to serious risk of bias, the quality of evidence overall for in-hospital mortality was classified as moderate, as the results of in-hospital mortality were robust and consistent, with NNT of 29 (95\% CI 23 to 40), no 

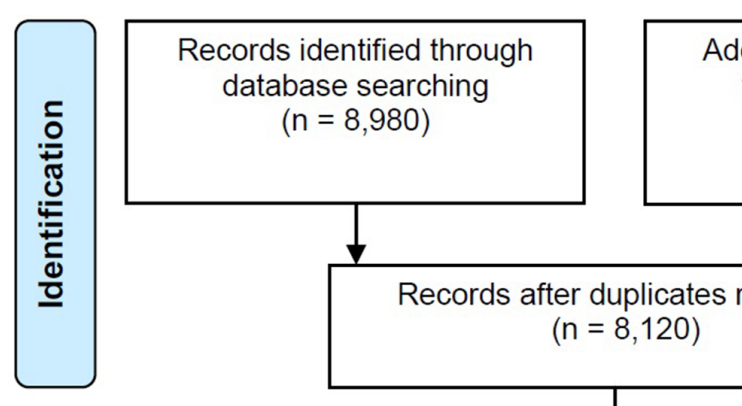

Additional records identified through other sources $(n=2,990)$
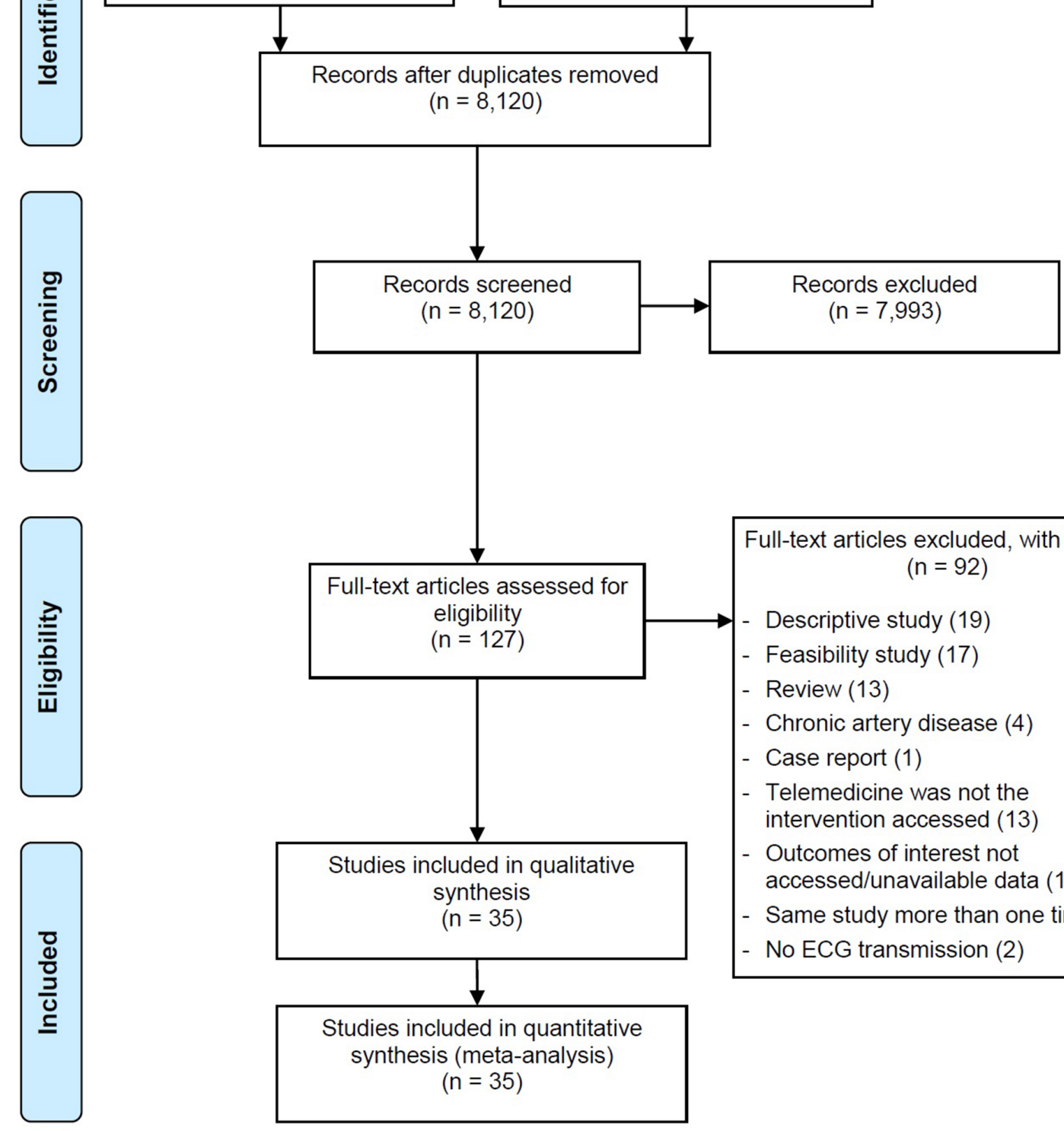

Full-text articles excluded, with reasons

$$
(\mathrm{n}=92)
$$

- Descriptive study (19)

- Feasibility study (17)

- Review (13)

- Chronic artery disease (4)

- Case report (1)

- Telemedicine was not the intervention accessed (13)

- Outcomes of interest not accessed/unavailable data (14)

- Same study more than one time (9)

- No ECG transmission (2)

Figure 1 Flow of information through the different phases of the systematic review. The flow chart was adapted from the Preferred Reporting Items for Systematic Review and Meta-Analyses flow chart model.

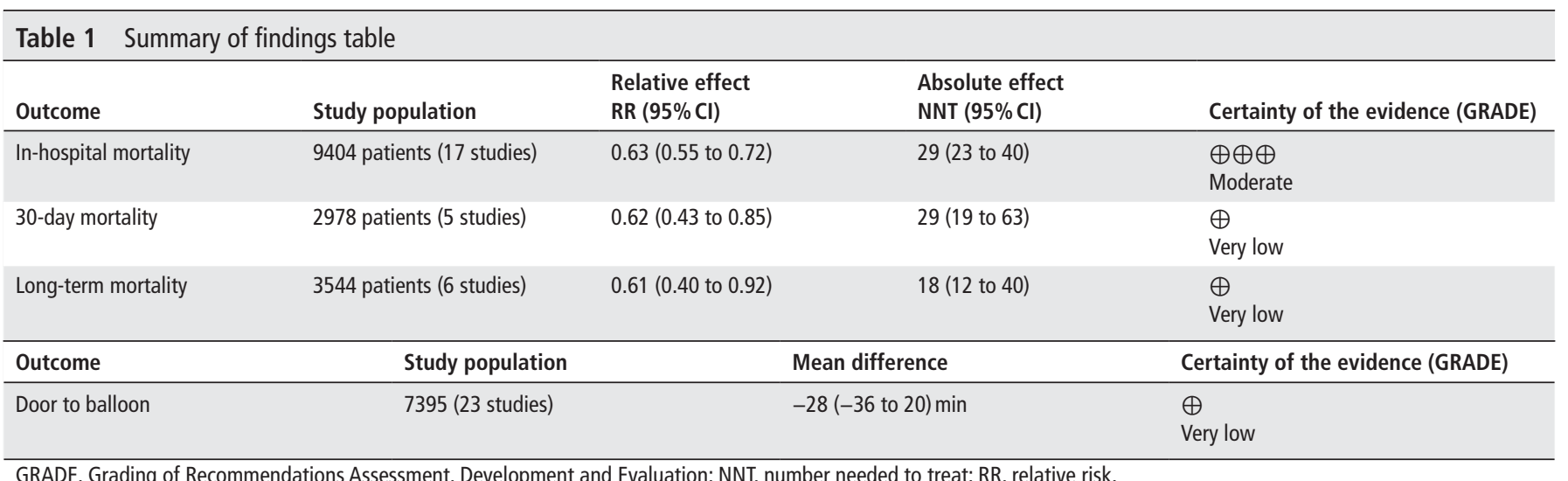




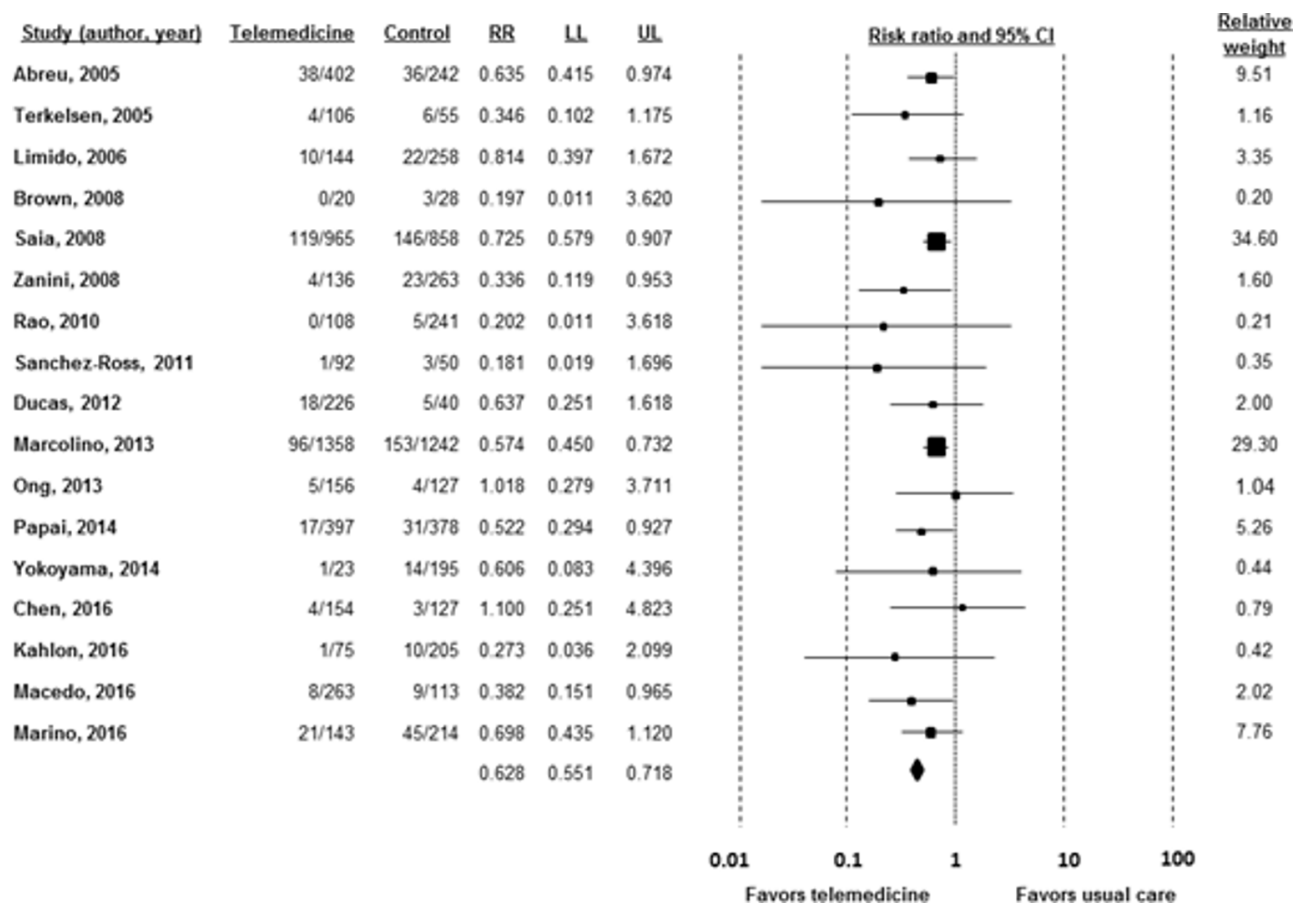

Figure 2 Forest plot showing the risk of in-hospital mortality in patients submitted to telemedicine strategies associated to usual care versus usual care. LL, lower limit; RR, relative risk; UL, upper limit.

evidence of heterogeneity and low risk of publication bias. There was also evidence of decreased 30-day mortality and long-term mortality. The low number of studies reporting these outcomes (5 and 6, respectively) precluded the formal assessment of publication bias, and concerns on heterogeneity and the risk of publication bias downgraded the evidence to very low. DTB time was reduced on average in $28 \mathrm{~min}$, with a precise $\mathrm{CI}$, despite the large heterogeneity that downgraded the evidence to very low.

There are challenges to implementation of telemedicine services, including barriers of cost and reimbursement, security of data, necessity to change existing workflows (and resistance to change) and in some areas lack of available high-speed bandwidth. However, this study supports development and maintenance of these systems despite these challenges, as it showed a clear impact in reducing AMI mortality.

Most of the publications selected in our comprehensive search used prehospital ECG transmission with ECG interpretation by a remote cardiologist or emergency physician for early diagnosis of STEMI and referral to PCI, with or without bypass of the emergency room. Another systematic review assessed the impact of the prehospital ECG, both transmitted for remote assessment and interpreted on-site, associated with advance notification of PCI team. It showed a positive impact in short-term mortality (in-hospital and 30-day mortality combined), with 39\% RR reduction in mortality over control (95\% CI 0.42 to 0.89$){ }^{26}$ This study included eight studies only and did not include any

\section{Funnel Plot of Standard Error by Log risk ratio}

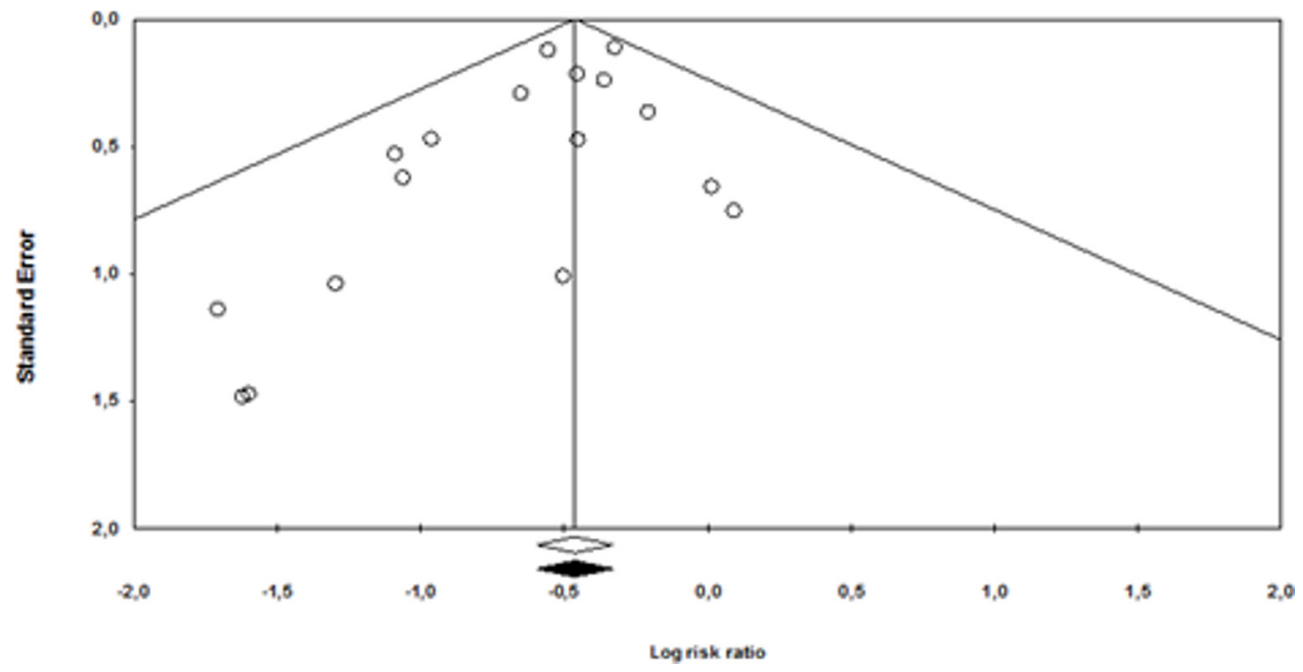

Figure 3 Funnel plot for the risk of in-hospital mortality. Observed studies are shown as open circles, and the observed point estimate is shown as open diamond. The imputed point estimate is shown as a filled diamond. As there is no imputed study, it is not different from the observed point estimate. 
$>$ 30-day mortality

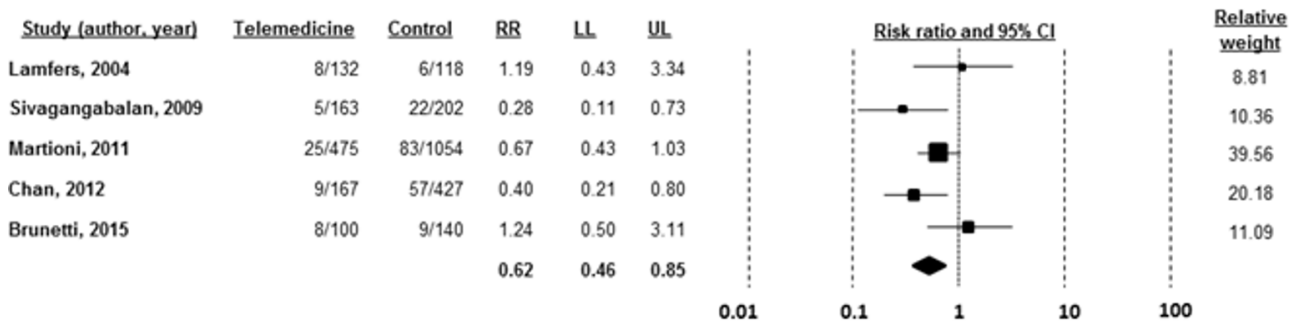

$>$ Long-term mortality

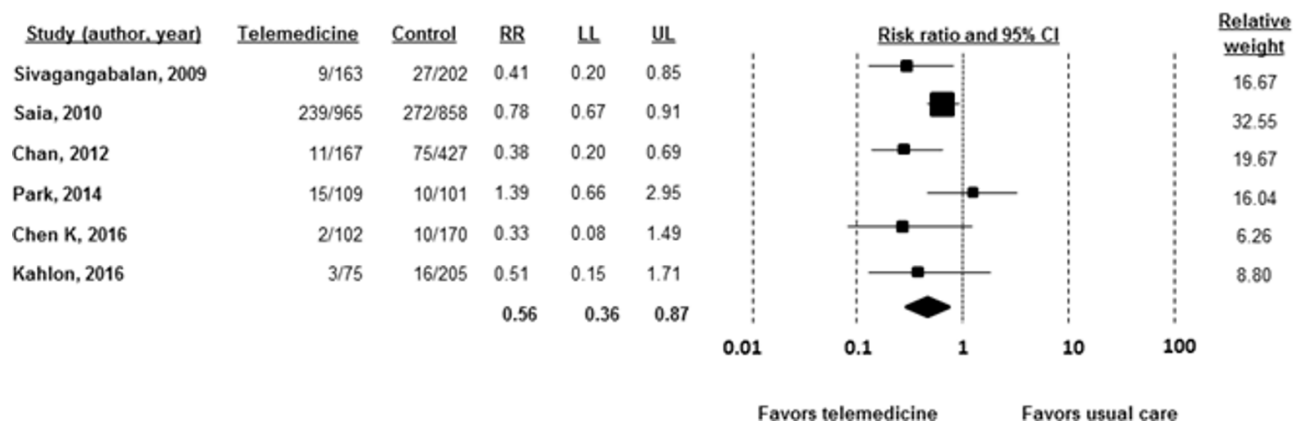

Figure 4 (A) Forest plot showing the risk of 30-day mortality in patients submitted to telemedicine strategies associated to usual care versus usual care. (B) Forest plot showing the risk of long-term mortality in patients submitted to telemedicine strategies associated to usual care versus usual care. LL, lower limit; RR, relative risk; UL, upper limit.

studies in low and middle income countries. Despite the fact that acquiring a prehospital ECG is a class I recommendation from the American Heart Association/American College of Cardiology, ${ }^{27}$ the experience of prehospital personnel in ECG interpretation varies. Therefore, our research question is of the utmost importance. Early diagnosis and treatment contribute to the observed reduction in mortality, but other factors might have positively impacted the mortality outcome. Telemedicine intervention is never isolated. It is always part of an integral strategy that includes training and motivation of healthcare professionals as well as higher integration between prehospital and in-hospital services, sometimes with direct patient admission in the catheterisation laboratory, bypassing the ED. Therefore, a combination of enhanced diagnostic accuracy of prehospital ECGs; access to

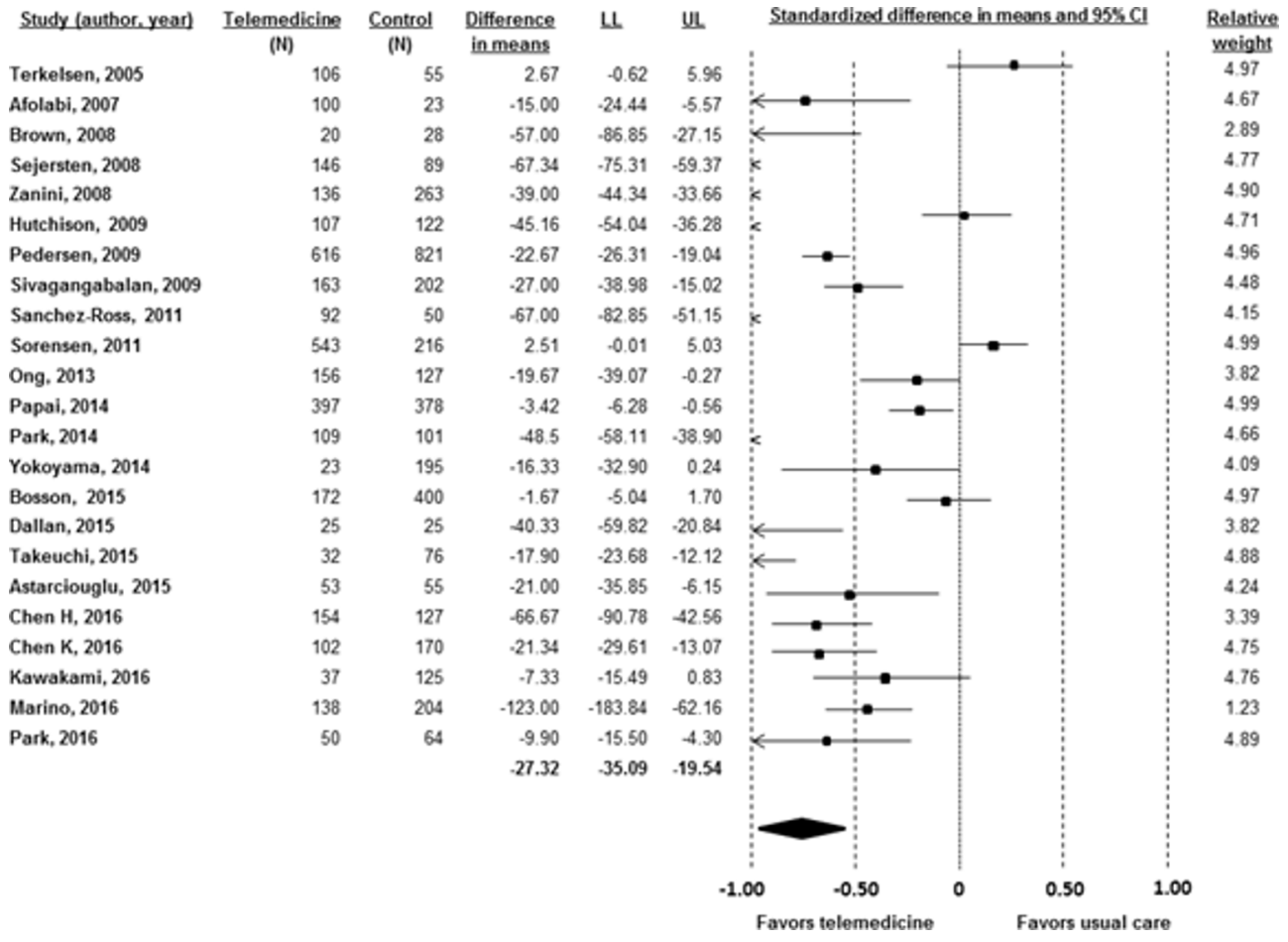

Figure 5 Forest plot showing the standardised mean difference for door-to-balloon time in patients submitted to telemedicine strategies associated to usual care versus usual care. LL, lower limit; RR, relative risk; UL, upper limit. 
specialised consultation by a distant cardiologist in the prehospital phase of care through teleconsultation; reduced treatment time by facilitating communication among professionals and, in some studies, direct transfer of patients to catheterisation laboratory; and improved quality of AMI treatment might be important contributors in the context of mortality outcome.

Despite the high levels of heterogeneity in the analyses comprising DTB time, telemedicine was associated with significant reduction in this time. The high heterogeneity may be due to the large SD of the means of reperfusion times reported in the studies. This could be explained by the long list of factors influencing the reperfusion time of AMI, such as the distance from the prehospital care location to the catheterisation laboratory, country and region of the study, presence of direct referral protocol to the catheterisation laboratory or passage through the emergency service, specificities of protocols for each centre; performance of the healthcare teams, and administrative and socioeconomic issues. ${ }^{28}$

Our data on DTB time corroborate with a recent meta-analysis that reported reduction of time to treatment achieved by prehospital triage with telemedicine. ${ }^{29}$ The study restricted the search to publications in English and in only one database, which resulted in the inclusion of only 11 studies, and one of them compared direct transport with intervention centre versus interhospital transport, both groups using telemedicine with no control without telemedicine. ${ }^{30}$

This review has limitations, as there were only observational studies and analysis by the ROBINS-I showed moderate to serious risk of bias, what impacts more in 30-day and longterm mortality results, because the paucity of the literature and the change of treatment strategy in the recent 10-20 years. The strength of our review is that it includes real-world studies, which incorporate typical barriers to implementation and other

\section{Key messages}

What is already known on this subject?

- Ischaemic heart disease remains the leading cause of death worldwide.

- Cardiology seems to be a promising area in telemedicine and, within cardiology, acute myocardial infarction care is one of the fields where most of the efforts have been made.

- The real impact of this intervention in clinical outcomes remains poorly documented as the results do not always show consistent positive impacts on clinical outcomes.

\section{What might this study add?}

- This systematic review and meta-analysis of 37 studies (16960 patients) found moderate-quality evidence that telemedicine strategies combined with usual care reduce in-hospital mortality (relative risk (RR) $0.63(95 \% \mathrm{Cl} 0.55$ to 0.72$)$ ), and very-low quality evidence that they reduce door-to-balloon time (mean difference $-28(95 \% \mathrm{Cl}$ -35 to -20$)$ min), 30-day mortality (RR $0.62(95 \% \mathrm{Cl} 0.43$ to 0.85$)$ ) and long-term mortality (RR $0.61(95 \% \mathrm{Cl} 0.40$ to $0.92))$.

\section{How might this impact on clinical practice?}

- This meta-analysis adds to the growing body of evidence supporting the use of telemedicine strategies, especially prehospital ECG transmission, to improve patient care in the management of patients with myocardial infarction. challenges that many systems face, for example, transmission failures or lack of adherence from the healthcare teams. This may make our results more generalisable.

In conclusion, this meta-analysis adds to the growing body of evidence supporting the use of telemedicine strategies, especially prehospital ECG transmission, to improve patient care in the management of patients with AMI. There is moderate quality evidence of significant reduction of in-hospital mortality and very low quality evidence of significant reduction in 30-day mortality, long-term mortality and DTB time.

Acknowledgements The authors thank Kuanchun Chen, Natale Daniele Brunneti, Robin Ducas and Tom Quinn for providing relevant information from their studies to be included in this research.

Contributors MSM and ALR conceived and planned the study. MSM, BLDP and LMM performed the search for relevant studies. Data were screened, extracted and analysed by LMM, JAQO and MSM. LDR and MSM were in charge of the quality assessment. LMM and MSM performed data analysis. MSM wrote the first draft of the paper. All authors contributed to the interpretation of the findings and critically revised it for intellectual content.

Funding This study was partially supported by grants of the Brazilian Research agencies Conselho Nacional de Desenvolvimento Cientifico e Tecnológico (CNPq), Fundação de Amparo a Pesquisa do Estado de Minas Gerais (FAPEMIG) and Coordenação de aperfeiçoamento de Pessoal de Nível Superior-Brasil (CAPES Finance code 001). ALR and MSM are members of the National Institute of Science and Technology for Health Technology Assessment (IATS/CNPq). The authors are solely responsible for the design and data analyses, the drafting and editing of the manuscript, and its final contents. The funders had no role in study design, data collection and analysis, decision to publish or preparation of the manuscript.

Disclaimer There are no financial competing interests related to this study. MSM, JAQO, LDR, ALR work in a public telemedicine service in Brazil. MSM and ALR participated in two projects related to the implementation of myocardial infarction system of care which included ECG transmission, respectively, in Belo Horizonte and in the Northern Region of Minas Gerais, Brazil. The publications were included in this systematic review; however, as mentioned in methods session, sensitivity analysis was performed, excluding those publications.

Competing interests None declared.

Patient consent for publication Not required.

Provenance and peer review Not commissioned; externally peer reviewed.

\section{REFERENCES}

1 Ibanez B, James S, Agewall S, et al. 2017 ESC Guidelines for the management of acute myocardial infarction in patients presenting with ST-segment elevation: The Task Force for the management of acute myocardial infarction in patients presenting with ST-segment elevation of the European Society of Cardiology (ESC). Eur Heart J 2017;39:119-77.

2 Blomkalns AL, Roe MT, Peterson ED, et al. Guideline implementation research: exploring the gap between evidence and practice in the CRUSADE Quality Improvement Initiative. Acad Emerg Med 2007;14:949-54.

3 Commission to the European Parliament, the Council. the European Economic and Social Committee and the Committee of the Regions on telemedicine for the benefit of patients, healthcare systems and society. Brussles, 2008. https://eur-lex.europa.eu/ legal-content/EN/ALL/?uri=CELEX:52008DC50689

4 Comission E, Commission E, Plan eHealthA. Innovative healthcare for the 21st century. Communication from the commission to the European parliament, the council, the European economic and social committee and the committee of the regions. 2012. Europe's place in a Global Race: High-Performance Computing, 2012-2020. https://eur-lex.europa.eu/legal-content/EN/TXT/PDF/?uri=CELEX: 52012DC50736\&from $=$ EN.

5 Brunetti ND, Scalvini S, Acquistapace F, et al. Telemedicine for cardiovascular disease continuum: A position paper from the Italian Society of Cardiology Working Group on Telecardiology and Informatics. Int J Cardiol 2015;184:452-8.

6 Caldarola P, Gulizia MM, Gabrielli D, et al. ANMCO/SIT consensus document: telemedicine for cardiovascular emergency networks. Eur Heart J Supp/ 2017:19:D229-D243.

7 Stroup DF, Berlin JA, Morton SC, et al. Meta-analysis of observational studies in epidemiology: a proposal for reporting. Meta-analysis Of Observational Studies in Epidemiology (MOOSE) group. JAMA 2000;283:2008-12.

8 Dissemination C. CRD's Guidance for Undertaking Reviews in Health Care. Layerthorpe: University of York, Centre for Reviews and Dissemination, 2009 
9 Higgins JPT CR, Chandler J, Cumpston MS, eds. Cochrane handbook for systematic reviews of interventions version 5.2.0 (updated June 2017), 2017. www.training. cochrane.org/handbook.

10 Moher D, Liberati A, Tetzlaff J, et al. Preferred reporting items for systematic reviews and meta-analyses: the PRISMA statement. Ann Intern Med 2009;151:264-9.

11 Nerlich M, Balas EA, Schall T, et al. Teleconsultation practice guidelines: report from G8 Global Health Applications Subproject 4. Telemed J E Health 2002;8:411-8.

12 Sterne JA, Hernán MA, Reeves BC, et al. ROBINS-I: a tool for assessing risk of bias in non-randomised studies of interventions. BMJ 2016;355:i4919.

13 Mentias A, Raza MQ, Barakat AF, et al. Effect of shorter door-to-balloon times over 20 years on outcomes of patients with anterior st-elevation myocardial infarction undergoing primary percutaneous coronary intervention. Am J Cardiol 2017;120:1254-9.

14 Guyatt GH, Oxman AD, Vist GE, et al. GRADE: an emerging consensus on rating quality of evidence and strength of recommendations. BMJ 2008;336:924-6.

15 Wan X, Wang W, Liu J, et al. Estimating the sample mean and standard deviation from the sample size, median, range and/or interquartile range. BMC Med Res Methodol 2014; $14: 135$

16 Lamfers EJ, Schut A, Hertzberger DP, et al. Prehospital versus hospital fibrinolytic therapy using automated versus cardiologist electrocardiographic diagnosis of myocardial infarction: abortion of myocardial infarction and unjustified fibrinolytic therapy. Am Heart J 2004;147:509-15.

17 Kawakami S, Tahara Y, Noguchi T, et al. Time to reperfusion in st-segment elevation myocardial infarction patients with vs. without pre-hospital mobile telemedicine 12lead electrocardiogram transmission. Circ J 2016;80:1624-33.

18 Sivagangabalan G, Ong AT, Narayan A, et al. Effect of prehospital triage on revascularization times, left ventricular function, and survival in patients with STelevation myocardial infarction. Am J Cardio/ 2009;103:907-12.

19 Brown JP, Mahmud E, Dunford JV, et al. Effect of prehospital 12-lead electrocardiogram on activation of the cardiac catheterization laboratory and doorto-balloon time in ST-segment elevation acute myocardial infarction. Am J Cardiol 2008;101:158-61.

20 Hutchison AW, Malaiapan Y, Jarvie I, et al. Prehospital 12-lead ECG to triage ST-elevation myocardial infarction and emergency department activation of the infarct team significantly improves door-to-balloon times: ambulance Victoria and MonashHEART Acute Myocardial Infarction (MonAMI) 12-lead ECG project. Circ Cardiovasc Interv 2009;2:528-34.

21 Strauss DG, Sprague PQ, Underhill K, et al. Paramedic transtelephonic communication to cardiologist of clinical and electrocardiographic assessment for rapid reperfusion of ST-elevation myocardial infarction. J Electrocardiol 2007;40:265-70.

22 Sterne JA, Egger M. Funnel plots for detecting bias in meta-analysis: guidelines on choice of axis. J Clin Epidemiol 2001;54:1046-55.

23 Duval S, Tweedie R. Trim and fill: A simple funnel-plot-based method of testing and adjusting for publication bias in meta-analysis. Biometrics 2000;56:455-63.

24 Saia F, Marrozzini C, Ortolani P, et al. Optimisation of therapeutic strategies for STsegment elevation acute myocardial infarction: the impact of a territorial network on reperfusion therapy and mortality. Heart 2009;95:370-6.

25 Saia F, Marrozzini C, Guastaroba P, et al. Lower long-term mortality within a regional system of care for ST-elevation myocardial infarction. Acute Card Care 2010;12:42-50.

26 Nam J, Caners K, Bowen JM, et al. Systematic review and meta-analysis of the benefits of out-of-hospital 12-lead ECG and advance notification in ST-segment elevation myocardial infarction patients. Ann Emerg Med 2014;64:176-86.

27 O'Gara PT, Kushner FG, Ascheim DD, et al. 2013 ACCF/AHA guideline for the management of ST-elevation myocardial infarction: a report of the American College of Cardiology Foundation/American Heart Association Task Force on Practice Guidelines. J Am Coll Cardiol 2013;61:e78-e140.

28 Perkins-Porras L, Whitehead DL, Strike PC, et al. Pre-hospital delay in patients with acute coronary syndrome: factors associated with patient decision time and home-tohospital delay. Eur J Cardiovasc Nurs 2009;8:26-33.

29 Brunetti ND, De Gennaro L, Correale M, et al. Pre-hospital electrocardiogram triage with telemedicine near halves time to treatment in STEMI: A metaanalysis and meta-regression analysis of non-randomized studies. Int J Cardiol 2017;232:5-11.

30 Dieker HJ, Liem SS, El Aidi H, et al. Pre-hospital triage for primary angioplasty: direct referral to the intervention center versus interhospital transport. JACC CardiovasC Interv 2010;3:705-11. 